\title{
STRICT VERIFICATION OF APPROXIMATE MIDCONVEXITY ON NON-CONVEX SETS
}

\author{
KRZYSZTOF MisZTAL AND JACEK TABOR
}

Abstract. Let $V$ be a subset of an Abelian group $G$ and let $\omega: V \times V \rightarrow[0, \infty]$ be given. We say that a function $f: V \rightarrow \mathbb{R}$ is $\omega(\cdot, \cdot)$-midconvex if

$$
f(x) \leqslant \frac{f(x-\delta)+f(x+\delta)}{2}+\omega(x-\delta, x+\delta)
$$

for $x \in V, \delta \in G$ such that $x-\delta, x+\delta \in V$. Our aim is to provide a computer assisted method to estimate

$$
\sup \{f \in V \rightarrow \mathbb{R}: f \in \mathscr{B}(V ; W), f \text { is } \omega(\cdot, \cdot) \text {-midconvex }\},
$$

where $\mathscr{B}(V ; W)$ denotes the set of real-valued, bounded from above functions on $V$ which are zero on $W(W \subset V)$. We present an algorithm which for given $\varepsilon>0$ enables us, under reasonable assumptions, to find the above supremum with accuracy $\varepsilon$. We test our results for $V=\left\{0, \frac{1}{N}, \ldots, \frac{N-1}{N}, 1\right\}$ and $W=\{0,1\}$, where $N \in \mathbb{N}$ is fixed.

Mathematics subject classification (2010): 26A51, 26B25.

Keywords and phrases: approximately convex function, approximately midconvex function, Jensen convexity, convexity on non-convex sets.

\section{REFERENCES}

[1] P. Allaart, K. KaWAmura, The Takagi function: a survey, preprint, 2011, available at http://arxiv.org/pdf/1110.1691.

[2] Z. Boros, An inequality for the Takagi functions, Math. Inequal. Appl. 11 (2008), 757-765.

[3] G. DAhlQUist, Å. BJÖRCK, Numerical methods in scientific computing, Society for Industrial and Applied Mathematics (2008), 263-264.

[4] D. H. Hyers, G. IsaC, Th. M. Rassias, Stability of Functional Equations in Several Variables, Birkhäuser, Basel, 1998.

[5] D. H. Hyers, S. M. Ulam, Approximately convex functions, Proc. Amer. Math. Soc. 3 (1952), $821-828$.

[6] Interval Arithmetics Library - ia_math: http://interval.sourceforge.net/interval/index.html.

[7] W. JARCZYK, M. LACZKOVICH, Almost convex functions on locally compact Abelian groups, Journal of Inequalities and Applications 13, 1 (2010), 217-225.

[8] W. JARCZYK, M. LACZKOvich, Convexity on abelian groups, Journal of Convex Analysis (2009), $33-48$.

[9] A. Házy, Zs. PÁLES, On approximately midconvex functions, Bull. London Math. Soc. 36 (2004) 339-350.

[10] J. MAKó, Zs. PÁLES, Approximate convexity of Takagi type function, J. Math. Anal. Appl. 369 (2010), $545-554$.

[11] R.E Moore, R.B. KeARFotT, M.J. Cloud, Introduction to interval analysis, Society for Industrial Mathematics (2009).

[12] NetBeans Pack for OpenGL Java Development:

http://netbeans-opengl-pack.dev.java.net/. 
[13] C. T. NG, K. Nikodem, On approximately convex functions, Proc. Amer. Math. Soc. 118 (1993), $103-108$.

[14] Zs. PÁLES, On approximately convex functions, Proc. Amer. Math. Soc. 131 (1) (2002), 243-252.

[15] M. Petković, L. Petković, Complex interval arithmetic and its applications, Wiley-VCH, 1998.

[16] JACEK TABOR, JÓZEF TABOR, Generalized approximate midconvexity, Control and Cybernetics 38 (2009), 655-669.

[17] JACEK TABOR, JÓZEF TABOR, Takagi functions and approximate midconvexity, JMAA 356 (2009), $729-737$. 